\title{
Genome-wide analysis of mutations in mutant lineages selected following fast-neutron irradiation mutagenesis of Arabidopsis thaliana
}

\author{
Eric J. Belfield, ${ }^{1}$ Xiangchao Gan, ${ }^{1,2}$ Aziz Mithani, ${ }^{1,8}$ Carly Brown, ${ }^{1}$ Caifu Jiang, ${ }^{1}$ \\ Keara Franklin, ${ }^{3}$ Elizabeth Alvey, ${ }^{4}$ Anjar Wibowo, ${ }^{1,5,9}$ Marko Jung, ${ }^{6}$ Kit Bailey, $^{1}$ \\ Sharan Kalwani, ${ }^{7,10}$ Jiannis Ragoussis, ${ }^{2}$ Richard Mott, ${ }^{2}$ and Nicholas P. Harberd ${ }^{1,11}$ \\ ${ }^{1}$ Department of Plant Sciences, University of Oxford, Oxford OX1 3RB, United Kingdom; ${ }^{2}$ Wellcome Trust Centre for Human Genetics, \\ University of Oxford, Oxford OX3 7BN, United Kingdom; ${ }^{3}$ School of Biological Sciences, University of Bristol, Bristol BS8 1 UG, United \\ Kingdom; ${ }^{4}$ Department of Plant Sciences, University of Cambridge, Cambridge CB2 3EA, United Kingdom; ${ }^{5}$ Center for Plant Stress \\ Genomics and Technology, King Abdullah University of Science and Technology, Thuwal 23955-690, Kingdom of Saudi Arabia; \\ ${ }^{6}$ Oxford e-Research Centre, University of Oxford, Oxford OX1 3QG, United Kingdom; ${ }^{7}$ Information Technology Department, King \\ Abdullah University of Science and Technology, Thuwal 23955-690, Kingdom of Saudi Arabia
}

\begin{abstract}
Ionizing radiation has long been known to induce heritable mutagenic change in DNA sequence. However, the genomewide effect of radiation is not well understood. Here we report the molecular properties and frequency of mutations in phenotypically selected mutant lines isolated following exposure of the genetic model flowering plant Arabidopsis thaliana to fast neutrons (FNs). Previous studies suggested that FNs predominantly induce deletions longer than a kilobase in A. thaliana. However, we found a higher frequency of single base substitution than deletion mutations. While the overall frequency and molecular spectrum of fast-neutron (FN)-induced single base substitutions differed substantially from those of "background" mutations arising spontaneously in laboratory-grown plants, G:C >A:T transitions were favored in both. We found that FN-induced G:C>A:T transitions were concentrated at pyrimidine dinucleotide sites, suggesting that FNs promote the formation of mutational covalent linkages between adjacent pyrimidine residues. In addition, we found that FNs induced more single base than large deletions, and that these single base deletions were possibly caused by replication slippage. Our observations provide an initial picture of the genome-wide molecular profile of mutations induced in $A$. thaliana by FN irradiation and are particularly informative of the nature and extent of genome-wide mutation in lines selected on the basis of mutant phenotypes from FN-mutagenized $A$. thaliana populations.
\end{abstract}

[Supplemental material is available for this article.]

Ionizing radiation is pervasive in the environment and acts as a natural mutagen via its DNA-damaging properties (Friedberg et al. 2006). In addition, the mutagenic property of artificial ionizing radiation has been a mainstay of genetic research since the pioneering experiments of Müller (1928). However, while the effects of ionizing radiation on individual genes are now relatively well understood, its genome-wide effects are not. We therefore undertook an analysis of the genome-wide consequences of fast neutron (FN) irradiation in Arabidopsis thaliana, comparing our findings with those of recent studies documenting the frequency and molecular spectrum of spontaneous "background" mutations in the genomes of laboratory-grown "mutation accumulation" (MA) line Arabidopsis plants (Ossowski et al. 2010) and of Arabidopsis plants regenerated in vitro from root tissue explants (Jiang et al. 2011). We found that exposure of Arabidopsis to FNs induces a broader range of mutational lesions than previously suspected, and

Present addresses: ${ }^{8}$ Department of Biology, LUMS School of Science and Engineering, Sector U-DHA, Lahore 54792, Pakistan; ${ }^{9}$ Department of Life Sciences, University of Warwick, Wellesbourne, Warwick CV35 9EF, UK; ${ }^{10} 1142$ Terra Ct., Rochester, MI 48306, USA.

${ }^{11}$ Corresponding author

E-mail nicholas.harberd@plants.ox.ac.uk

Article published online before print. Article, supplemental material, and publication date are at http://www.genome.org/cgi/doi/10.1101/gr.131474.111. that both the incidence and spectrum of FN-induced mutations are distinct from those of "spontaneous" mutations. These discoveries have important consequences for the use of FNs in experimental plant mutagenesis and provide an indication of the likely mutagenic effects of environmental ionizing radiation on organisms living in the wild (Hinton et al. 2007).

\section{Results}

FN irradiation, mutant generation, and isolation

Our analyses began with a multiply mutant Arabidopsis line [predominantly Landsberg erecta (Ler) background carrying the mutations gai-t6, rga-t2, rgl1-1, rgl2-1, and rgl3-4; henceforth called progenitor] (see Methods) that lacks the growth-regulating DELLA proteins. Progenitor $\left(\mathrm{M}_{1}\right)$ seeds were exposed to FN irradiation (see Methods), with the long-term aim (although not the focus of this study) of isolating and characterizing novel growth-regulation mutants in a DELLA-deficient genetic background. Accordingly, $\mathrm{M}_{2}$ seedlings were screened for elongated hypocotyls, a mutant phenotype in which exaggerated growth of the hypocotyl (embryonic stem structure) results in a longer hypocotyl than that of progenitor controls. This class of mutant commonly contains loss-of-function mutations in genes that 
mediate the light inhibition of hypocotyl growth (Chen et al. 2004). We identified more than 300 independent putative elongated hypocotyl mutants and selected six of these (E71, E99, E125, E128, $\mathrm{E} 138$, and E216) to confirm heritability and for analysis in subsequent generations.

\section{Creation of a progenitor reference sequence and alignment of progenitor reads to Col-0 reference}

To identify mutations in the genomes of the six selected elongated hypocotyl mutants, we first needed to obtain a wholegenome reference sequence of the progenitor from which they were derived. We therefore obtained Illumina Genome Analyzer DNA sequence data from a single progenitor line plant and used these data to create a progenitor reference genome (see Methods). Initial mapping of the progenitor paired-end sequencing reads to the Arabidopsis Information Resource (TAIR 9) (http://www. arabidopsis.org) Col-0 reference genome (using MAQ v0.7.1) (Li et al. 2008) revealed single nucleotide polymorphisms (SNPs) and insertions and deletions (INDELs). This observation is consistent with the fact that only $3.2 \mathrm{Mb}$ of the $\sim 120-\mathrm{Mb}$ progenitor genome is derived from Col-0 (Fig. 1), while the remaining $\sim 97 \%$ of the genome is of Ler origin. To eliminate the progenitor versus TAIR 9 variants observed and to aid the identification of the genetic lesions caused by FN mutagenesis, we created our own progenitor reference genome sequence (see Methods).

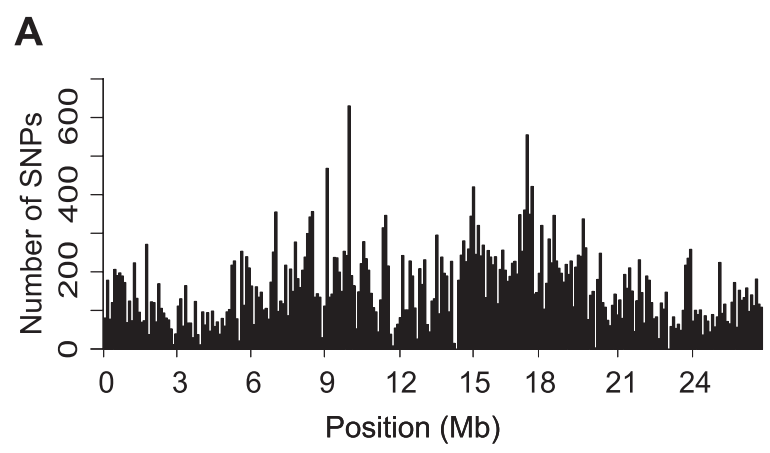

B

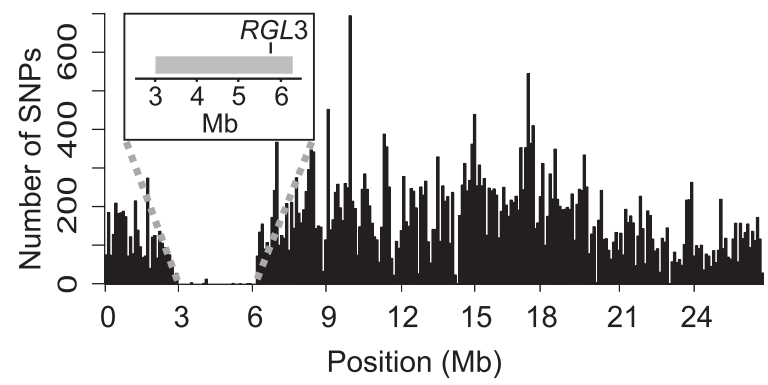

Figure 1. Density of single nucleotide polymorphisms (SNPs) detected following alignment of $(A) A$. thaliana Landsberg erecta (Gan et al. 2011) and $(B)$ progenitor reads to the Col-0 (TAIR 9) reference genome sequence. Histograms of SNPs along the length of chromosome 5 are shown. The Col-0 segment of progenitor chromosome 5 (containing the rg/3-4 T-DNA insertion) is clearly visible in (B), between $3 \mathrm{Mb}$ and $6.25 \mathrm{Mb}$, where the number of variants (versus TAIR 9) falls close to baseline. The site of the RGL3 locus is shown in the Col-0 DNA segment (in gray; see inset).

\section{Detection of mutations in FN-induced mutant lineages}

We next generated Illumina Genome Analyzer DNA sequence data from single $\mathrm{M}_{3}$ plants homozygous for the phenotype-conferring mutations in the six selected mutant lineages. We were able to identify a near-complete set of homozygous DNA sequence variants (versus the progenitor reference) in the nonrepetitive fractions of these mutant genomes. A mutation (base substitution or INDEL) was called if $95 \%-100 \%$ of aligned reads in a sample differed from the progenitor reference sequence (see Methods). We detected a total of 108 homozygous mutations (see Fig. 2; Table 1; Supplemental Table 1). Of the 56 variants detected in lines E99 and $\mathrm{E} 125$, all but one was independently confirmed by direct PCR amplification and Sanger sequencing, indicating that our falsepositive discovery rate was negligible. The unconfirmed variant in E125 was a G:C>A:T substitution that was difficult to confirm because it lies in a region of sequence that is represented repetitively in the Arabidopsis genome.

\section{Estimation of original numbers of mutations in FN-irradiated plants}

While the above 108 homozygous mutations were detected in $\mathrm{M}_{3}$ plant genomes, they had originally been induced by FN irradiation as heterozygous mutations in $\mathrm{M}_{1}$ plant genomes (Fig. 3). We therefore calculated the original mutation numbers in the following way. Assuming recessivity of the initial elongated hypocotyl mutation, each original $\mathrm{M}_{2}$ mutant seedling would have been homozygous for the mutation causing that phenotype (and any neighboring mutations) (see Fig. 3). In addition, there was an approximately one-fourth chance of the original $\mathrm{M}_{2}$ mutant seedling being homozygous for induced mutations (of unknown phenotypic consequence) in other regions of the genome. These regions of "fixed" homozygosity would have been uniformly transmitted to the subsequent $\mathrm{M}_{3}$ generation. Further mutations, heterozygous in the original $\mathrm{M}_{2}$ seedling, will have been segregating in $\mathrm{M}_{3}$ seedlings (the genome of one of which was sequenced). Thus, of the total 108 detected homozygous $\mathrm{M}_{3}$ mutations (Supplemental Table 1), 72 were likely homozygous in the $M_{2}$, with the remaining quarter (36) resulting from an estimated 144 variants that had been heterozygous in the $\mathrm{M}_{2}$ and had then become homozygous in the $\mathrm{M}_{3}$ (see Fig. 3). Mutations heterozygous in the $\mathrm{M}_{3}$ generation were overwhelmingly likely to have been discarded from variant lists (and thus not detected) because of failure to reach the 95\%-100\% read difference criterion described above. In summary, we estimate that the 108 homozygous mutations detected in $\mathrm{M}_{3}$ plants (Fig. 2) imply an original number of $\sim 288$ heterozygous FN-induced mutations in $\mathrm{M}_{1}$ plants (Fig. 3).

\section{Detection of spontaneous mutations in the progenitor line}

The progenitor line lacks the five growth-regulating DELLA proteins and is substantially derived from the Ler genetic background. To be sure that the mutations detected in E71, E99, E125, E128, E138, and E216 were genuinely FN-induced and were not simply of spontaneous origin, we determined the frequency of spontaneous mutation in the progenitor line. We sequenced the genomes of single plants from three independent control progenitor lineages. Each of these three plants $\left(\mathrm{F}_{2}-1\right.$ to -3$)$ was an $\mathrm{F}_{2}$ generation plant (the product of two successive self-pollination generations) derived from a control, nonirradiated progenitor plant (and hence the control equivalent of the $\mathrm{M}_{2}$ ) (Fig. 3). Using our above-described 
A

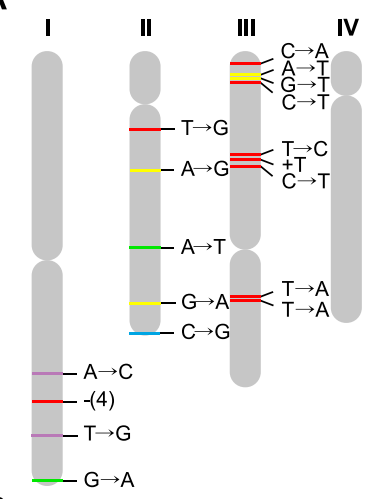

C

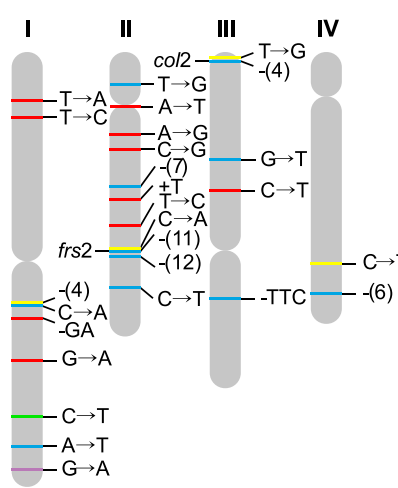

E
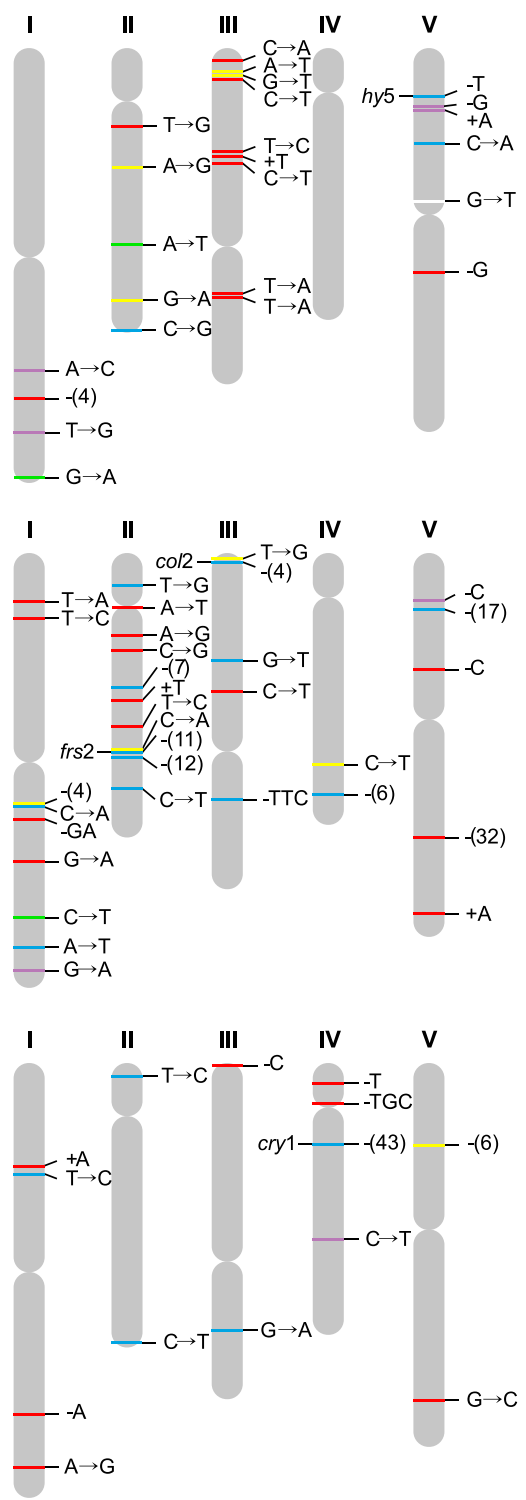

B

D

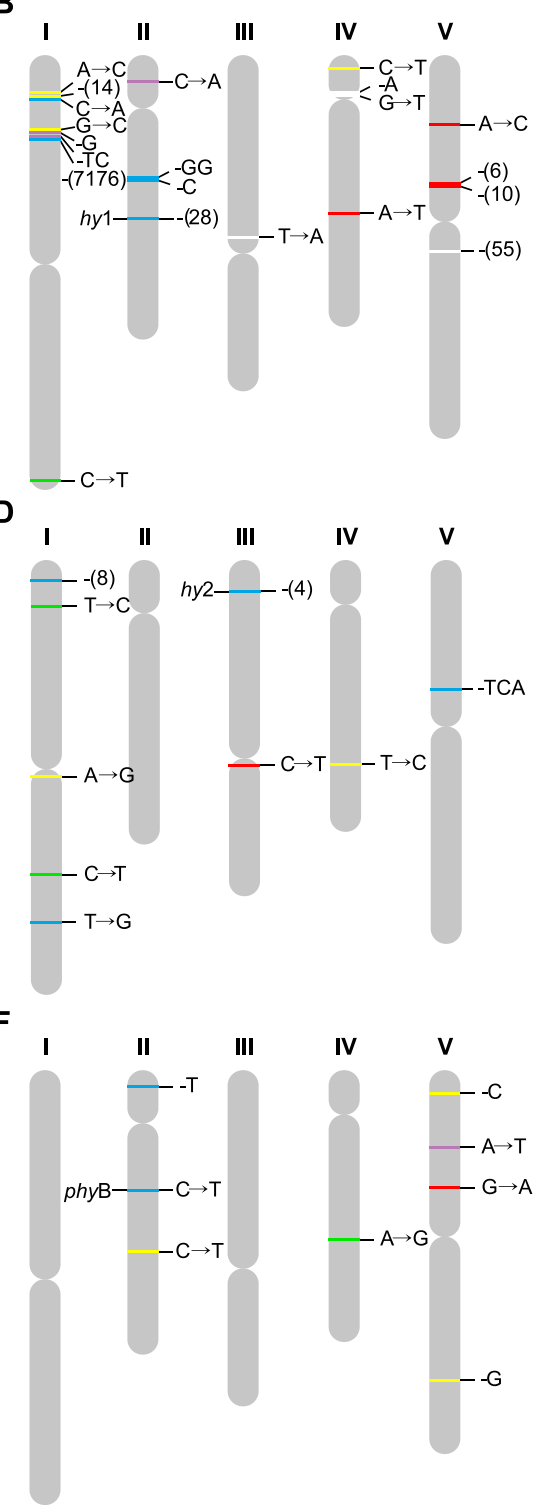

$\mathbf{F}$

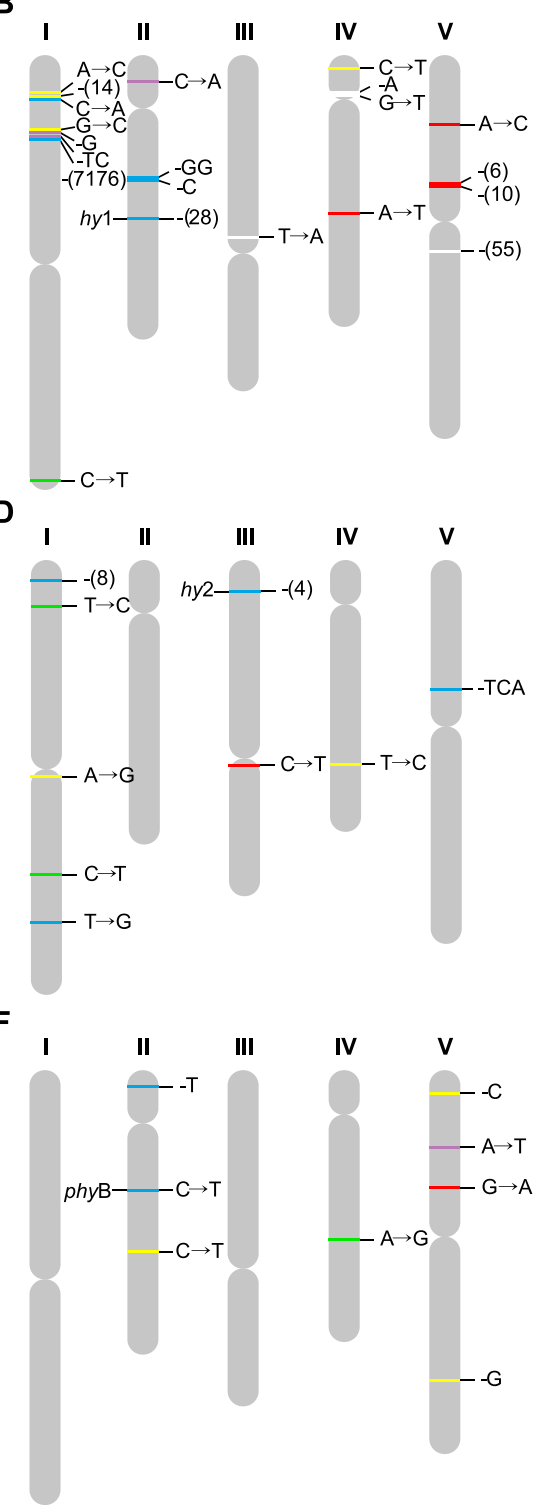

a total of seven previously undetected mutations were additionally detected in the E99 and E125 M data sets (Supplemental Table 3). Because these additionally detected mutations may have become detectable (homozygous) in the $\mathrm{M}_{4}$ having been undetectable (heterozygous) in the $\mathrm{M}_{3}$, we used Mendelian segregation laws (as in Fig. 3) to predict that there should have been 8.8 such newly detected homozygous variants, a figure slightly higher than the number (seven) actually observed (Supplemental Fig. 1; Supplemental Tables 3, 4). Thus spontaneous mutations do not significantly inflate the number of detected mutations in $\mathrm{M}_{4}$ plants beyond that predicted on the basis of Mendelian segregation of FN-induced heterozygous mutations in $\mathrm{M}_{3}$ plants.

These two separate control experiments indicate that the frequency of spontaneous mutations in the multiple DELLA knockout progenitor line is comparable to that previously observed in the Col-0 wild-type (WT) line (Ossowski et al. 2010). Because the frequency of mutations in FN-irradiated lineages (Figs. 2, 3) is one to two orders of magnitude higher than in the control lineages, we conclude that the mutations detected in our FN-irradiated lineages are indeed substantially caused by FN irradiation.

\section{Chromosomal distribution and frequencies of $\mathrm{FN}$-induced mutations}

The 108 detected FN-induced mutations (Fig. 2) ranged in size from single base substitutions (SBSs) and insertions to deletions of one or two bases, to larger deletions ( 3 bases to $1 \mathrm{~kb}$ or greater) (Table 1 ; Supplemental Table 1). The distribution of mutations across chromosomes in each of the six lineages is shown in Figure 2. The number of mutations per line ranged from eight to 32 (Fig. 2; Table 1). These differences are significant (G-test, $P=$ $1.7 \times 10^{-4}$ ) and may reflect heterogeneity in radiation dosage or in water content of irradiated seeds (seed radiosensitivity is dependent on water content) (Kamra et al. 1960).

FN exposure increases both overall

mutation detection methods, we identified only one single mutation in these three genomes (an A:T>C:G substitution in the $\mathrm{F}_{2}-2$ line) (Supplemental Table 2).

In a separate determination of the frequency of spontaneous mutation in the progenitor, we sequenced the genomes of two individual $\mathrm{M}_{4}$ plants (progeny of E99 and E125 genome-sequenced $\mathrm{M}_{3}$ lines) and compared the mutations detected in the $\mathrm{M}_{3}$ with those detected in the $\mathrm{M}_{4}$ (Supplemental Fig. 1). While all mutations previously detected in the $\mathrm{M}_{3}$ were also detected in the $\mathrm{M}_{4}$, mutation frequency and the frequency of specific molecular mutation classes. Taking all detected single base mutations together (SBSs and INDELs), we estimated the mutation rate to be $359.7 \times 10^{-9}$ per site in the irradiated generation following correction for underlying spontaneous mutations (Table 2A; Supplemental Table 5). In essence, a 60-Gy FN dose caused a transient single-generational $\sim 50$-fold increase in mutation rate above the spontaneous rate $\left(7.1 \times 10^{-9}\right.$ per site per generation) (Ossowski et al. 2010). 
Table 1. Mutations identified in six FN-irradiated $M_{3}$ plant lineages

\begin{tabular}{|c|c|c|c|c|c|c|c|c|}
\hline Mutation & E71 & E99 & E125 & E128 & E138 & E216 & Total & Percentage \\
\hline Substitutions & 18 & 10 & 18 & 6 & 7 & 5 & 64 & 100.0 \\
\hline Coding & 4 & 2 & 6 & 3 & 4 & 3 & 22 & 34.4 \\
\hline Silent & 2 & 1 & 1 & 2 & 0 & 1 & 7 & 10.9 \\
\hline Missense & 2 & 1 & 5 & 1 & 4 & 0 & 13 & 20.3 \\
\hline Truncating & 0 & 0 & 0 & 0 & 0 & 1 & 1 & 1.6 \\
\hline Untranslated region & 2 & 1 & 1 & 0 & 1 & 1 & 6 & 9.4 \\
\hline Intronic & 4 & 3 & 3 & 2 & 0 & 0 & 12 & 18.8 \\
\hline Splice site affected & 0 & 0 & 0 & 1 & 0 & 0 & 1 & 1.6 \\
\hline Splice site unaffected & 4 & 3 & 3 & 1 & 0 & 1 & 12 & 18.8 \\
\hline Intergenic & 7 & 2 & 8 & 1 & 2 & 1 & 21 & 32.8 \\
\hline Transposable element & 1 & 2 & 0 & 0 & 0 & 0 & 3 & 4.7 \\
\hline Insertions & 2 & 0 & 2 & 0 & 1 & 0 & 5 & 100.0 \\
\hline Coding & 0 & 0 & 0 & 0 & 0 & 0 & 0 & 0 \\
\hline Untranslated region & 1 & 0 & 0 & 0 & 0 & 0 & 1 & 20.0 \\
\hline Intronic $^{\mathrm{a}}$ & 0 & 0 & 0 & 0 & 0 & 0 & 0 & 0 \\
\hline Intergenic & 1 & 0 & 2 & 0 & 1 & 0 & 4 & 80.0 \\
\hline Transposable element & 0 & 0 & 0 & 0 & 0 & 0 & 0 & 0 \\
\hline Deletions & 4 & 11 & 12 & 3 & 6 & 3 & 39 & 100.0 \\
\hline Coding & 1 & 4 & 7 & 3 & 1 & 1 & 17 & 40.5 \\
\hline Untranslated region & 1 & 2 & 1 & 0 & 0 & 0 & 4 & 9.5 \\
\hline Intronic $^{\mathrm{a}}$ & 0 & 1 & 1 & 0 & 1 & 2 & 5 & 11.9 \\
\hline Intergenic & 2 & 2 & 3 & 0 & 4 & 0 & 11 & 26.2 \\
\hline Transposable element & 0 & 2 & 0 & 0 & 0 & 0 & 2 & 4.8 \\
\hline
\end{tabular}

Spectrum and number of homozygous mutations inferred by BWA and IGV analyses and their distributions among functional classes in each of six FN M 3 mutant lines (E71, E99, E125, E128, E138, and E216).

${ }^{\mathrm{a}}$ Intronic splice site unaffected.

On a genome-wide scale, $35 \%$ of the 108 detected FN mutations were in genic regions (see Supplemental Fig. 2), which is higher than the expected 33\% but not significant (G-test, $P=0.6$ ). This observation suggests that mutations are not preferentially located in gene-coding, gene regulatory, or intergenic regions (Fig. 2; Supplemental Table 1), despite previous indications of mutational bias associated with chromatin structural difference (Prendergast et al. 2007) and the possibility that local differences in chromatin architecture might differentially affect DNA repair mechanisms, and hence localized mutation rate.

\section{FN-induced INDEL mutations}

Although FN exposure was previously thought to induce a predominance of deletion mutations in plants (Bruggemann et al. 1996; Li et al. 2001), we, in fact, detected substantial impact on the relative frequencies of a range of specific mutational classes. Among INDELs, we detected 19 large deletions ( $>3 \mathrm{bp}$ ), ranging in size from $4 \mathrm{bp}$ to $7.2 \mathrm{~kb}$, and six smaller deletions (2-3 bp) (Fig. $4 \mathrm{~A}$ ) in our FN-exposed lineages. In addition, we detected 14 single base deletions, making this the most prominent class of deletion mutation detected (Fig. 4A), unlike previous suggestions that 2- to 4 -kb deletions are the most frequent class of FN-induced deletion mutation (Bruggemann et al. 1996; Li et al. 2001). We also detected five single base insertions. The latter were all A or T insertions, suggesting a possible bias against $\mathrm{G}$ or $\mathrm{C}$ insertions and consistent with an apparent bias in favor of A:T composition (Table 2B) as previously reported in Col-0 MA lines (Ossowski et al. 2010).

Single base deletion and insertion mutations are often caused by replication slippage at homopolymer or polynucleotide repeat regions (Viguera et al. 2001). Consistent with this, of the 19 FN-induced single base INDELs, all five insertions and nine of the
14 (64\%) deletions either occurred within or were adjacent to homopolymeric or polynucleotide repeats (Table $3 \mathrm{~A}$ ). In addition, six of the seven 3-bp or 4-bp deletions were located within adenine or thymine homopolymeric or polynucleotide repeats (although larger FNinduced deletions appear less likely to be associated with mononucleotide or dinucleotide microsatellites; seven of 15 ; 47\%) (Table 3B). These observations suggest the possibility that ionizing radiation may promote the incidence of DNA replication slippage.

Ionizing radiation frequently generates gross chromosomal aberration in plants (interstitial inversions, translocations, etc.) (Shikazono et al. 2005). To identify such events, we searched for "distant-pair" signatures (as previously described) (Korbel et al. 2007; Gan et al. 2011; Jiang et al. 2011), where a pair of reads from either end of a genome sequencing fragment that straddles a "breakpoint" (inversion or translocation chromosomal aberration) with respect to the progenitor reference genome align at an unexpected distance apart on that reference. Despite exhaustive searches, we did not detect any distant pairs, indicating the absence of gross chromosomal aberration in our six post-irradiation lineages. However, analysis of a further FN-induced $\mathrm{M}_{3}$ mutant line, E88 (full mutation profile not included in this study), detected a chromosomal rearrangement mutation (an interstitial inversion of $\sim 215 \mathrm{~kb}$ ), thus indicating that our methods are robust (Supplemental Fig. 3A). In addition, using our standard methods, an $\sim 21$-kb deletion (larger than observed in the original six $\mathrm{FN} \mathrm{M}_{3}$ lines) was identified in the $\mathrm{E} 8 \mathrm{M}_{3}$ line (Supplemental Fig. 3B).

\section{FN-induced single base substitutions}

Exposure to FNs also induced a relatively high frequency of single base substitutions (SBSs). SBSs can be transitions (purine > purine; pyrimidine $>$ pyrimidine) or transversions (purine > pyrimidine; pyrimidine $>$ purine), and FNs induced both types of substitution (Fig. 4B,C; Supplemental Tables 1, 6). C>T transitions were the most common substitution in MA lines (Fig. 4B; Ossowski et al. 2010), consistent with $C$ being the most spontaneously mutable base, and were also prominent in FN-exposed lineages (Fig. 4C). Another form of radiation, UV, is known to induce the formation of covalent linkages between adjacent pyrimidine residues (where a $\mathrm{C}$ or $\mathrm{T}$ is adjacent to another $\mathrm{C}$ or $\mathrm{T}$ ) in DNA sequence, resulting in a predominance of $\mathrm{UV}$-induced $\mathrm{C}>\mathrm{T}$ mutations at dipyrimidine sequences (Daya-Grosjean and Sarasin 2005). We identified 64 FNinduced SBSs of which $51(80 \%)$ were at pyrimidine dinucleotide sites, which is more than expected by chance (Fisher's exact test, $\left.P=1.9 \times 10^{-6}\right)($ Supplemental Tables 1, 6). Of these SBSs, 20 were $\mathrm{C}>\mathrm{T}$ mutations and 14 of these $(70 \%)$ were at pyrimidine dinucleotide sites (Supplemental Table 6); this was not found to be significant (Fisher's exact test, $P=0.1$ ). We conclude that FN exposure induces $\mathrm{C}>\mathrm{T}$ transitions (and other SBSs) via sequence context-dependent mechanisms preferentially targeting pyrimi- 


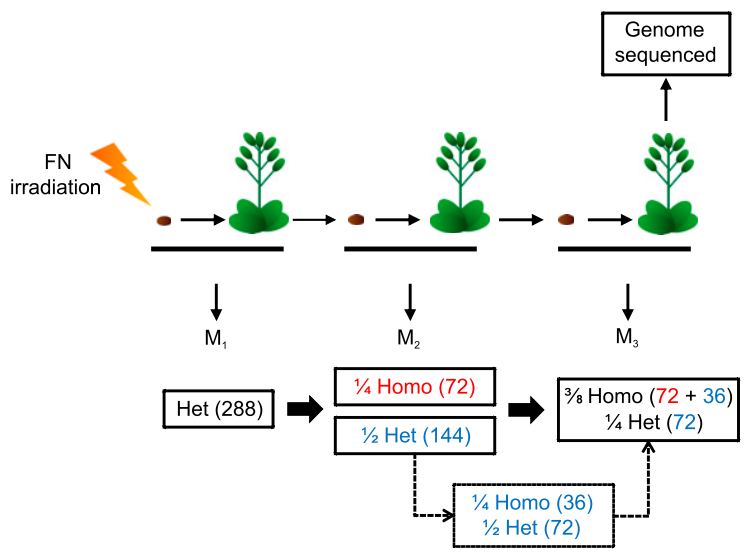

Figure 3. Estimation of original numbers of mutations in $M_{1}$ plants. $\mathrm{M}_{1}$ seeds were irradiated with $\mathrm{FNs}$, germinated, and resultant plants selfpollinated to yield $M_{2}$ seed. Elongated hypocotyl mutants were identified in the $M_{2}$ and self-pollinated to yield $M_{3}$ seed. The genomes of six mutant $M_{3}$ plants were sequenced. Mendelian segregation laws were used to estimate the number of variants, both homozygous (Homo) and heterozygous (Het), in the generations preceding the six genome-sequenced $\mathrm{FN} \mathrm{M}_{3}$ mutant lines. As shown, all homozygous mutations "fixed" in the $\mathrm{M}_{2}$ (in red) will have remained homozygous in the $\mathrm{M}_{3}$. In addition, a quarter of $\mathrm{M}_{2}$ heterozygous mutations (144; blue text) will have segregated in a Mendelian 1:2:1 (homozygous mutation:heterozygous mutation:homozygous nonmutant) ratio, with approximately one-quarter becoming homozygous mutations ( 36 ; blue text) in the $M_{3}$. Thus, a total of 108 homozygous variants (detected by our methods) along with a further predicted 72 segregating heterozygous variants (not detected by our methods) are estimated for the $M_{3}$. To summarize, in the original $\mathrm{FN}$-exposed $\mathrm{M}_{1}$ plants, a total of 288 (black text) heterozygous mutations are estimated to have arisen. One-quarter of these mutations will have become homozygous in the $M_{2}$ (72; red text), half becoming heterozygous (144; blue text), with the remaining quarter being homozygous nonmutant. These considerations permit calculation (see Supplemental Table 5) of FN-induced mutation rates.

dine dinucleotides. While previously associated particularly with UV exposure, our results suggest that pyrimidine dinucleotideassociated $\mathrm{C}>\mathrm{T}$ transitions are actually a feature of exposure to a wider range of radiation classes.

Another prominent class of FN-induced substitution was the G>T transversion (see Supplemental Table 1). Radiation-induced DNA damage is often attributed to the effects of reactive oxygen species (ROS) and hydroxyl radicals generated when water molecules absorb high-energy particles. In particular, ROS are thought to damage guanine residues, thus precipitating $\mathrm{G}>\mathrm{T}$ mutations (Kawanishi et al. 2001). Our observation of a relatively high frequency of these transversions is thus consistent with these arising through FN-induced ROS-mediated DNA damage.

Overall, the FN-induced SBS mutational spectrum is very different from that of spontaneous SBSs seen in MA lines (Fig. 4B,C; Ossowski et al. 2010). Transversions are relatively more frequent in FN-induced than in spontaneous SBSs, resulting in a much reduced transition/transversion (Ti/Tv) ratio ( $\sim 1$ for $\mathrm{FN}$-induced mutations versus $\sim 3$ for spontaneous mutations) (Fig. 4B,C). Interestingly, a relatively low $\mathrm{Ti} / \mathrm{Tv}$ ratio is also characteristic of mutations observed in in vitro regenerant plant lineages (Jiang et al. 2011).

Mutations in the six $\mathrm{FN} \mathrm{M}_{3}$ lines likely to confer the elongated hypocotyl phenotype

In each of the six selected $\mathrm{M}_{3}$ lines, we were able to identify the mutation that likely confers the mutant elongated hypocotyl phenotype (mutations in genes HY1, HY2, HY5, FRS2, COL2, CRY1, and $P H Y B$; see below) (Fig. 2). In each line a within-gene deletion or nonsynonymous mutation (unique to that line) presumably confers mutant phenotype, with all other mutations likely having no significant effect on the phenotype. Bioinformatic analyses of the six FN elongated hypocotyl mutant lineages indicated that the likely phenotypic causal mutations were as follows: E71: a 1-bp deletion in an exon of HY5 (a gene encoding a bZIP transcription factor that is a positive regulator of photomorphogenesis) (Ang et al. 1998); E99: a 28-bp deletion that spans part of the promoter, start codon, and first exon of $H Y 1$ (a gene that encodes a plastid heme oxygenase necessary for phytochrome chromophore biosynthesis) (Davis et al. 1999); E125: an 11-bp deletion in an exon of FRS (a gene that encodes a protein essential for phytochrome A-controlled far-red light responses) (Lin and Wang 2004) and a 4-bp deletion in an exon of COL2 (a gene that encodes a zinc finger protein with $\sim 67 \%$ amino acid identity to the protein encoded by the flowering-time gene CONSTANS) (Ledger et al. 2001); E128: a 4-bp deletion in HY2 (which like HY1, is necessary for phytochrome chromophore biosynthesis) (Kohchi et al. 2001); E138: a 43-bp deletion in CRY1 (a gene that encodes a cryptochrome blue light

Table 2. Comparison of FN-exposure lineage versus MA line mutation rates

A. Mutation rates per site in both Col-0 MA lines (Ossowski et al. 2010) and FN-induced mutant lineages (E71, E99, E125, E128, E1 38, and E216)

\begin{tabular}{lcrr}
\hline & \multicolumn{2}{c}{$\begin{array}{c}\text { Mutation rate } \\
\text { per site }\left(\times \mathbf{1 0}^{-\mathbf{9}}\right)\end{array}$} & \\
\cline { 2 - 3 } Mutation & MA lines & FN lines & $\begin{array}{c}\text { Fold-rate } \\
\text { difference }\end{array}$ \\
\hline Substitutions & 6.5 & 264.6 & 40.7 \\
Single base deletions & 0.3 & 74.4 & 248.0 \\
Single base insertions & 0.3 & 20.7 & 69.0 \\
All mutations & $\mathbf{7 . 1}$ & $\mathbf{3 5 9 . 7}$ & $\mathbf{5 0 . 7}$
\end{tabular}

B. A comparison of mutation rates per genome per generation calculated from Col-0 MA lines versus FN lines ${ }^{a}$

\begin{tabular}{lccccc}
\hline & \multicolumn{2}{c}{ MA lines } & & \multicolumn{2}{c}{$\begin{array}{c}\text { FN lines } \\
\text { (minus spontaneous rate) }\end{array}$} \\
\cline { 2 - 3 } \cline { 5 - 6 } Mutation & Rate & SEM & & Rate & SEM \\
\hline Deletions & 0.06 & 0.02 & & 6.32 & 1.01 \\
Large deletions (>3 bp) & 0.03 & 0.01 & & 3.09 & 0.71 \\
Single base deletions & 0.03 & 0.01 & & 2.23 & 0.60 \\
A & 0.02 & 0.01 & & 0.27 & 0.19 \\
T & 0.01 & 0.01 & & 0.48 & 0.28 \\
G & 0 & 0 & & 0.67 & 0.33 \\
C & 0.01 & 0.01 & & 0.81 & 0.36 \\
Single base insertions & 0.03 & 0.01 & & 0.73 & 0.33 \\
A & 0.01 & 0.01 & & 0.48 & 0.28 \\
T & 0.03 & 0.01 & & 0.25 & 0.18 \\
G & 0 & 0 & & 0 & 0 \\
C & 0 & 0 & & 0 & 0 \\
Single base substitutions & 0.66 & 0.07 & & 1.76 & 0.51 \\
A:T>G:C & 0.08 & 0.02 & & 2.17 & 0.49 \\
G:C $>$ A:T & 0.39 & 0.05 & & 1.19 & 0.42 \\
A:T>C:G & 0.05 & 0.02 & & 1.57 & 0.50 \\
A:T>T:A & 0.03 & 0.01 & & 1.49 & 0.47 \\
G:C $>$ T:A & 0.06 & 0.02 & & 0.51 & 0.25 \\
G:C $>$ C: $:$ S & 0.05 & 0.02 & & 8.69 & 1.09 \\
\hline
\end{tabular}

${ }^{a}$ The FN line data were corrected with the MA line data to account for underlying spontaneous mutation rates.

Totals for all mutations are shown in bold. Standard errors of the means (SEM) are shown.

\section{Genome Research www.genome.org}


A

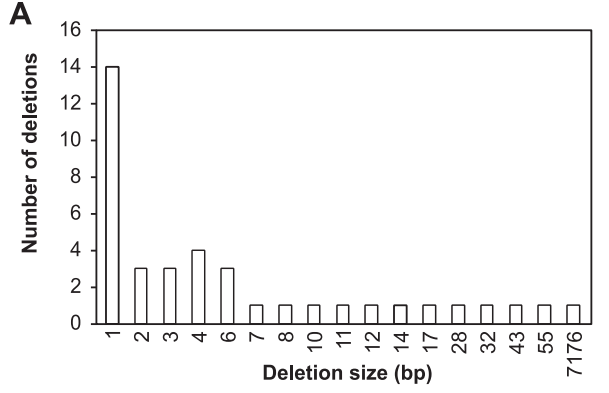

B
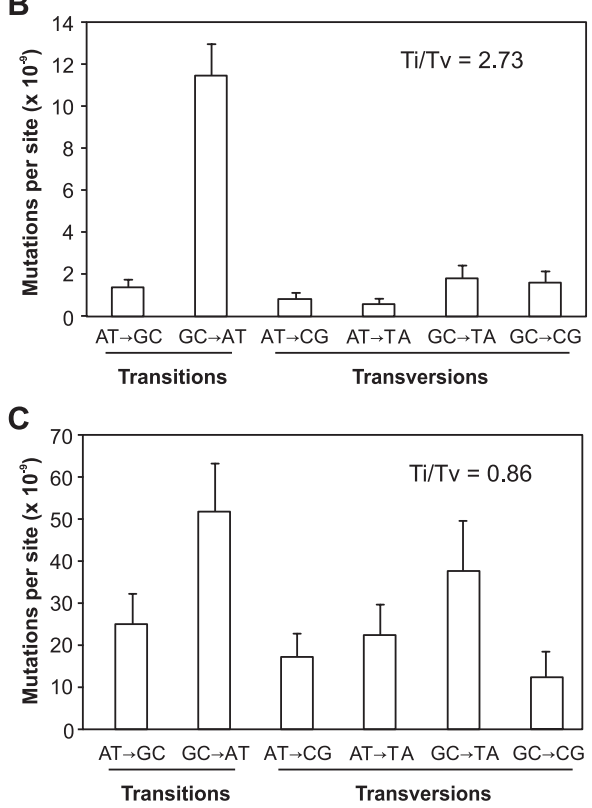

Figure 4. Molecular spectrum of $\mathrm{FN}$-induced mutations. ( $A$ ) Frequencies and sizes of deletion mutations identified in the six FN-exposed mutant lineages. $(B, C)$ SBS rates per A:T or $\mathrm{G}: \mathrm{C}$ site and transition/transversion (Ti/Tv) ratios in (B) Col-0 MA lines (Lynch 2010; Ossowski et al. 2010) and (C) FN-exposed lineages. Complementary mutations, e.g., $\mathrm{A}>\mathrm{C}$ and $\mathrm{T}>\mathrm{G}$, are pooled. The Ti/Tv ratio for each profile is shown. The mutation rate is the mutation rate weighted by the incidence of the base in the Arabidopsis Col-0 genome.

photoreceptor) (Ahmad et al. 1998); and E216: a 1-bp nucleotide substitution in an exon of PHYB (a gene encoding a phytochrome red light photoreceptor) (Somers et al. 1991).

\section{Discussion}

This study reveals for the first time the genome-wide consequences of irradiation on plant genomes, and the spectrum and rate of mutations that researchers should expect to find in irradiationmutagenized populations. In contrast to previous reports, we find that FN-induced single base substitutions (SBSs) are more prevalent than FN-induced INDELs (Table 1), and that short deletions $<3 \mathrm{bp}$ are more prevalent than larger-scale deletions (Fig. 4A). Among FNinduced SBSs, we detected a bias toward G:C >A:T transitions at dipyrimidine sites and a higher overall frequency of FN-induced transversions versus transitions (with respect to spontaneous SBSs found in MA lines) (Fig. 4B,C).

Overall, the molecular mutational spectrum of FN-induced SBSs is distinct from that of the spontaneous SBSs found in MA lines (Fig. 4B,C). Since MA lines are grown in conditions of relative shelter from environmental mutagenic agents, it is likely that any differences in mutational spectra reflect exposure to a mutagenic environment (in this case, a FN bombardment beam), and imply that the increased frequency of mutations due to FN exposure is not simply due to an accelerated occurrence of the same classes of spontaneous mutations as found in MA lines. There are similarities between the characteristics of FN-induced SBSs and those observed in in vitro regenerant Arabidopsis plants (Jiang et al. 2011), although why this should be the case is not clear.

FN irradiation of plants is traditionally considered to induce deletion mutations of 2-4 kb in size (Bruggemann et al. 1996; Li et al. 2001) and has been used for many years in forward genetics experiments to determine the function of unknown genes (Hoffmann et al. 2007; Hofer et al. 2009). An alternative mutagen is the alkylating agent ethylmethane sulphonate (EMS). While EMS mutagenesis produces both complete and partial loss-of-function mutant alleles (Bowman et al. 1991), almost exclusively via G:C>A:T point mutations (Greene et al. 2003; Martin et al. 2009) at frequencies of $\sim 1000$ mutations per Arabidopsis genome (Jander et al. 2003; Martin et al. 2009), FN mutagenesis was previously thought to produce full loss-of-function alleles by inducing a range of deletions (300 bp to $8 \mathrm{~kb}$ ) (Bruggemann et al. 1996) and insertions ( 3.4 kb) (Sun et al. 1992). However, our identification of 108 FN-induced genomic molecular variants indicate that these previous studies may have favored the detection of large-scale mutations. Of the 39 deletions that we detected, $38(97 \%)$ were $<56$ bp in length, with single base deletions being the most frequently observed $(36 \%$, 14 of 39) (Table 3) and with the majority of single base deletions (64\%, nine of 14) occurring at homopolymeric or dinucleotide sites (Table 3A). Thus the view that FNs predominantly induce relatively large-scale gene-inactivating deletion mutations seems less likely, although we cannot exclude the possibility that some larger deletions (up to megabases), which often have a reduced male transmission rate (Naito et al. 2005) or may cause lethality when homozygous, may have been under-represented in our genomesequenced $\mathrm{M}_{3}$ lines. Indeed, analysis of an additional FN-induced mutant line, E88, did confirm that FNs can induce larger deletions (than those observed in the original six $\mathrm{FN} \mathrm{M}_{3}$ lines) of $>20 \mathrm{~kb}$ (see Supplemental Fig. 3B), and in addition, a chromosomal rearrangement mutation (an interstitial inversion of $\sim 215 \mathrm{~kb}$ ) was identified (see Supplemental Fig. 3A). Taken together, our findings suggest that the six FN lineages analyzed in this study provide a good overall picture of the kinds of variants caused by FNs, although the picture may be biased in favor of mutations less likely to have deleterious effects.

We have here described the establishment of six elongated hypocotyl mutant lineages from mutant homozygotes identified in the $\mathrm{M}_{2}$ generation. In each case, we were able to identify the likely phenotype-conferring mutation (see Fig. 2). However, a major previous concern in mutant screens of this nature has been the unknown frequency, chromosomal distribution, and phenotypic consequence of additional mutagen-induced mutations. This concern is the basis of the traditional approach of sequential backcrossing of novel phenotype-causal mutant alleles to the nonmutagenized progenitor, with the aim of segregating out unknown additional mutations (although mutations genetically linked to the phenotype-causal allele will tend to be retained). Because we analyzed the genomes of lineages selected on the basis of mutant phenotype, it is possible that the number of detected additional mutations is somewhat different from what would have been found in unselected $\mathrm{M}_{3}$ plants. However, our results show for the first time that additional mutations, although they 
Table 3. Genome-wide analysis of radiation-induced mutations in six $\mathrm{FN} \mathrm{M}_{3}$ mutant lines A

\begin{tabular}{|c|c|c|c|}
\hline Line & Loci & $\begin{array}{l}\text { INDEL size } \\
\text { (bp) }\end{array}$ & Sequence \\
\hline E138 & Chr1:7137500 & +1 & AGAAAAAAAGAAAAAAAAAAA \\
\hline E125 & Chr2:10220586 & +1 & CAGGGATAGCTTTTTTTTTTT \\
\hline E71 & Chr3:7761881 & +1 & AАTTTTTTTG \\
\hline E125 & Chr5:25236520 & +1 & GCAAATCTTGAAAAAAAAAAA \\
\hline E71 & Chr5:4105018 & +1 & CCCAGACTATAAAGGTTCGA \\
\hline E138 & Chr1:24646357 & -1 & AAACAAAATCAAAATTTAATA \\
\hline E99 & Chr1:5288028 & -1 & TACTGCTGTTGTTCGATCAGT \\
\hline E216 & Chr2:1354114 & -1 & GAAAGATGACTCTACAAGCCA \\
\hline E99 & Chr2:8735139 & -1 & GTGGATCGGTCTTATTTAGGG \\
\hline E138 & Chr3:267625 & -1 & AAAGTGCACTCATTATCCCTC \\
\hline E138 & Chr4:1885738 & -1 & TTCTGAATGGTTAGAATAGG \\
\hline E99 & Chr4:3109274 & -1 & CGCGGAACCTAGAGGAGAACC \\
\hline E71 & Chr5:15965759 & -1 & GATTTTCTAG \\
\hline E216 & Chr5:1930240 & -1 & ATCTGTAAATCTTTTTACAGG \\
\hline E216 & Chr5:22317677 & -1 & TAATTGGTTGGCTCTTATACA \\
\hline E125 & Chr5:3438719 & -1 & GTGAGATTGACAGTTCGGTGC \\
\hline E71 & Chr5:3594216 & -1 & СTCTCGCTGCTTGATGGTAAA \\
\hline E71 & Chr5:4105012 & -1 & TTTCTGCCCAGACTATAAGGG \\
\hline E125 & Chr5:8315155 & -1 & GTTCGGTAACCAAAATTTATA \\
\hline
\end{tabular}

B

\begin{tabular}{l|l|c|l}
\hline Line & \multicolumn{1}{|c|}{ Loci } & $\begin{array}{c}\text { Deletion size } \\
\text { (bp) }\end{array}$ & \multicolumn{1}{c}{ Sequence } \\
\hline E125 & Chr3:17229771 & -3 & TCCTCTCTTTTTCTCTGTTTCA \\
E138 & Chr4:2999448 & -3 & TTGTTCTTAATGCTAGTTCTAGA \\
E128 & Chr5:7998106 & -3 & GAGCTGTTTTCATGGAATATGT \\
E125 & Chr1:17747994 & -4 & GAAAATAATTAATAAAATAGCAC \\
E71 & Chr1:24826965 & -4 & GGCTTTGGAAAAATTCCTCTAATT \\
E128 & Chr3:2804349 & -4 & CCCATGGGGAGGGAATTGACTGG \\
E125 & Chr3:487491 & -4 & CCTTGATCCGTGGTCTTATCTCTG \\
E125 & Chr4:16632942 & -6 & GTTGGAGAAACTAACTCTAAACAAGA \\
E138 & Chr5:5256590 & -6 & AAGAACCAGTGACCAAATAACTTCCT \\
E99 & Chr5:9194110 & -6 & ATTACCAACTTACCATCGGACACAAA \\
E125 & Chr2:9135557 & -7 & CTATGTGCGTAAGAAGCAAGCTTTCTC \\
E128 & Chr1:2199360 & -8 & GAGTCCGATCCAAACCCTAGGTTAGCCG \\
E99 & Chr5:9469535 & -10 & AATATGTTCGGATAATGGATTGACGAGTTA \\
E125 & Chr2:13691669 & -11 & TTCTACAGAGAATATGCTCGATCAGTGGGAT \\
E125 & Chr2:13804210 & -12 & CAGGAACCAATACAGAGCTAGCTATAGCTAAT \\
E99 & Chr1:2896321 & -14 & ATGCACCAGGGTAAGACAACACCAGTAAAGGCTA \\
E125 & Chr5:3727272 & -17 & ATCCCATGACAAGCCATGGCCAAAATTAGGGCTAATT \\
E99 & Chr2:11340050 & -28 & CTGTAACCATTTTCTA. . TGGCGTATTTAGCTCCG \\
E125 & Chr5:19925087 & -32 & TGTTTTGAACATGAAGA. . TATGAACGTACACGTAA \\
E138 & Chr4:5723251 & -43 & TGAGAGATGTCTGGTTC . . GTATTGTATTGTATGGT \\
E99 & Chr5:13714219 & -55 & CTTAGTCGATGCAGGAG . . AGAAGATCATAGAGGCG \\
E99 & Chr1:6220669 & -7176 & AGTTTGAACTTTTAAT . . ACAGCAATGCACACGCA \\
& & &
\end{tabular}

INDEL mutation locations and flanking sequences of (A) single base INDELs and (B) larger deletion mutations. Homopolymeric and polynucleotide stretches are shown underlined. Bases in bold are those that were inserted or deleted.

clearly occur, are not as frequent as might have been supposed, and that the traditional backcrossing approach is indeed a relatively effective means of "cleaning up" the genetic background of mutant lineages. Most importantly, our results identify the genome-wide spectrum of mutations that researchers can expect to find as additional mutations in mutants selected from FN-irradiation mutagenized material.

Our observations have consequences for the use of FN irradiation as a mutagen in plant genetic studies, including pub- licly available resources such as those for Arabidopsis (http://www.

lehleseeds.com), soybean (http://www. soybase.org), and rice (http://www.ars. usda.gov). They indicate the spectrum of mutations that researchers can expect when using FN irradiation in reverse genetic screens. The high frequency of single base variants suggests that likely causal mutations may not be as readily identifiable using traditional deletion-detection approaches as previously supposed. In addition, our results provide an initial picture of the genome-wide mutational effect of exposure to ionizing radiation, identifying in particular an increase in the relative frequency of transversion mutations. The presence in the environment of cosmic radiation and natural and artificial radionuclides implies a consequential radiation exposure of all organisms. Genome-wide DNA sequence analysis can now be used as a measure of previous exposure to ionizing radiation. Next-generation sequencing of plant genomes could contribute to our understanding of the effect and consequences of environmental ionizing radiation on natural plant populations, thus helping in the implementation of effective environmental protective measures (Copplestone et al. 2010).

Our analyses reveal for the first time the genome-wide consequences of FN irradiation on plant genomes and the spectrum and rate of mutations that researchers should expect to find in FNmutagenized plant populations. In addition, our study provides a valuable benchmark for future studies of the effects of ionizing radiation on genome integrity.

\section{Methods}

\section{Plant material}

All experiments used the Landsberg erecta (Ler) laboratory strain of $A$. thaliana as genetic background. The progenitor "global-DELLA" (gai-t6, rga-t2, rgl1-1, rgl2-1, and rgl3-4) line was isolated from the $F_{3}$ progeny of a cross between Ler "quadruple-DELLA" (gai-t6, rga-t2, rgl1-1, and rgl2-1) (Achard et al. 2006) and a line carrying a T-DNA insertion in the $A$. thaliana Columbia0 RGL3 locus ( $r g l 3-4)$ obtained from the publicly available SAIL (Syngenta Arabidopsis Insertion Lines) collection. This rgl3-4 line had previously been backcrossed six times sequentially to the Ler background.

\section{Plant mutagenesis, growth conditions, and mutant screening}

Progenitor seeds were mutagenized by FN bombardment at a dose of $60 \mathrm{~Gy}$ at the KFKI Atomic Energy Research Institute (Budapest,

\section{Genome Research} www.genome.org 
Hungary) and then sown on soil in glasshouses with a 16-h light/ 8 -h dark photoperiod at $22^{\circ} \mathrm{C}-24^{\circ} \mathrm{C}$ (irradiance $120 \mu \mathrm{mol}$ of photons $/ \mathrm{m}^{2}$ per second). The resultant $\mathrm{M}_{1}$ plants were allowed to self-pollinate in pools of about 1000 plants. $\mathrm{M}_{2}$ and $\mathrm{M}_{3}$ plants were grown in controlled environment rooms (CERs) with a 16-h light/8-h dark photoperiod at $22^{\circ} \mathrm{C}-24^{\circ} \mathrm{C}$ (irradiance $120 \mu \mathrm{mol}$ of photons $/ \mathrm{m}^{2}$ per second).

Genetic screens of $\mathrm{M}_{2}$ seedlings for elongated hypocotyl mutants were performed by sowing seeds on soil in CERs and allowing them to germinate and grow for 5-7 d. Seedlings were screened visually for elongated hypocotyls. From a total of about 400,000 seeds, more than 300 mutant seedlings displaying elongated hypocotyls were isolated, grown, and self-pollinated. Segregation analysis based on hypocotyl length was performed on 40 $\mathrm{M}_{3}$ plants per line, identifying homozygous lines E71, E88, E99, E125, E128, E138, and E216.

\section{Genome sequencing}

Young leaf samples were taken from single nonirradiated progenitor $\mathrm{F}_{2}$, or $\mathrm{FN} \mathrm{M}_{3}$ and $\mathrm{M}_{4}$ mutants as well as progenitor plants. DNA was isolated using a Plant DNeasy Mini kit (QIAGEN). Samples representing three independent nonirradiated progenitor derived $F_{2}$ lines $\left(F_{2}-1\right.$ to -3 ; control plants equivalent to the $M_{2}$ generation in Fig. 3), seven elongated hypocotyl $\mathrm{M}_{3}$ mutant lines (E71, E88, E99, E125, E128, E138, and E216), an individual E99 $\mathrm{M}_{4}$ and an E125 $\mathrm{M}_{4}$ mutant plant, and the progenitor itself were sequenced using the Illumina Genome Analyzer II platform according to the manufacturer's instructions at the Wellcome Trust Center for Human Genetics, Oxford, UK, GeneServices, UK or the Beijing Genomics Institute, China. Several lanes of 36-, 51-, 75-, 76-, 90-, and/or 101-bp paired-end runs were produced for each line. A 24-85 (raw sequencing data) and 17-58 (aligned sequencing data) fold depth sequence coverage was obtained for each sample (Supplemental Table 7; sequencing data statistics for the E88 $\mathrm{M}_{3}$ line are not shown).

\section{Creation of progenitor reference sequence}

To identify DNA sequence variants in the genomes of the six FNirradiated $\mathrm{M}_{3}$ mutant lineages (E71, E99, E125, E128, E138, and E216; versus progenitor), it was necessary to create our own reference genome. We developed a software package called Iterative Read-Mapping and Realignment (IMR) v0.3.0 (http://mus.well. ox.ac.uk/19genomes/IMR-DENOM/) (Gan et al. 2011), which was used to create the progenitor genome reference sequence. The algorithm underlying IMR iteratively modifies and corrects the reference, such that in each iteration, sequencing reads are mapped to the current version of the reference using MAQ v0.7.1 (Li et al. 2008) and variants identified with high confidence are used to update and improve the reference sequence. The reads are then remapped and the process repeated until convergence. For our data five iterations were run.

In each iteration base substitution calling is performed using an algorithm based on SAMtools v0.1.5c varFilter (Li et al. 2009). The default behavior of varFilter (which we follow) is to accept isolated base substitutions (with less than two other variants within $10 \mathrm{bp}$ ) if:

(1) coverage $\geq 3$ and $<100$; and

(2) the root mean square (RMS) of the mapping qualities (Phred scores) of the aligned reads $>25$.

Short INDELs (up to $30 \mathrm{bp}$ ) reported by SAMtools (Li et al. 2009) were accepted. Under certain circumstances, SAMtools reported two different deletions at the same location. In such cases, the more likely deletion, usually the longer, was accepted.
One important issue that the IMR algorithm addresses is the problem of clusters of base substitutions and whether these are genuine or artifacts of undetected INDELs (often the alignments of the ends of sequencing reads generate base substitution calls in preference to INDELs). Since varFilter of SAMtools (Li et al. 2009) automatically filters out variant sites where three or more base substitutions occur within $10 \mathrm{bp}$, these cases were reconsidered as follows: the base substitutions with the greatest confidence (i.e., highest RMS) were accepted, and the two criteria (1) and (2) above were applied. Sites that passed these criteria were modified. Variants that were close to INDELs (i.e., within $10 \mathrm{bp}$ ) were ignored since they would be considered in the next iteration.

We also found a number of sites that could not be fixed with IMR because there were two or more different bases in the progenitor sequencing reads aligning to one position in the Col-0 reference sequence. To overcome this, we developed a filter to call base substitutions at these ambiguous sites in the progenitor whereby a base substitution was called at a position if:

(1) at least $80 \%$ of reads had the same base;

(2) the number of sequencing reads was within $\pm 20 \%$ of the average genome-wide read coverage.

In total, 169.5 million filtered paired-end progenitor reads were aligned to the original Col-0 reference sequence to create our own progenitor genome reference sequence of $119.6 \mathrm{Mb}$.

\section{Detection of DNA sequence variants}

The bioinformatic methods used in this study are the same as those used in Jiang et al. (2011), which found that neither data type nor method significantly altered observed relative frequency or spectrum of detected single base substitutions (SBSs) and small INDELs. In addition, the systematic comparisons performed in Jiang et al. (2011) showed that differences in types of data and data analysis methods between our methods and those used in Ossowski et al. (2010) had negligible effect on detected SBSs and INDELs, and that false-negative rates would have been at comparable small levels in both cases. These observations are also consistent with a recent publication indicating that different softwares are in general comparable (Lunter and Goodson 2011).

Sequencing reads from the three control nonirradiated plants that were derived from the progenitor and grown in independent lineages over two successive generations $\left(\mathrm{F}_{2}-1\right.$ to -3$)$, seven FN-induced elongated hypocotyl mutants (E71, E88, E99, E125, E128, E138, and E216; $\mathrm{M}_{3}$ generation), and two $\mathrm{M}_{4}$ generation E99 and E125 plants were next mapped to the progenitor reference genome using the Burrows-Wheeler Aligner (BWA) v 0.5.8a (Li and Durbin 2010). Reads with Phred-scaled mapping quality of 20 or less, reads that were not uniquely mapped, and reads for which the mate (the other read of a pair) did not map onto the reference genome were excluded from further analyses. Between 106.6 and 107.9 million nucleotide sites in the six $\mathrm{FN} \mathrm{M}_{3}$ lines (E71, E99, E125, E128, E138, and E216) had sufficient read information (at least one read) for calling initial variants compared with the progenitor reference (an average of 107.5 million sites was used to determine the mutation rates per site calculated in Table 2).

The variant lists were generated for each data set using SAMtools (Li et al. 2009) after alignment with the progenitor reference genome sequence. The variant lists were then filtered to exclude putative false positives called at sites with low sequencing read coverage (that could lead to miscalling errors) or high coverage (that are associated with DNA sequences with a high degree of similarity to other sequences in the genome such as transposable elements). A minimum of seven and a maximum of 75 sequencing reads per site were set as the base sub- 
stitution threshold limits. For INDELs, at least two reads per site were required. Furthermore, base substitution and INDEL false positives called by SAMtools (Li et al. 2009) after re-alignment of the progenitor sequencing reads to the progenitor reference genome sequence using BWA were subtracted from our elongated hypocotyl mutant variant lists.

Following the filtering processes above, the base substitutions and INDEL lists were then checked manually by visually scanning the alignment files (BAM files) generated by SAMtools (Li et al. 2009) using a locally customized version of Integrated Genome Viewer (IGV) (Robinson et al. 2011), which allowed visual comparison of all aligned reads for each mutant data set with the progenitor reference. The six lines analyzed in this study were simultaneously visualized in IGV (for an example, see Supplemental Fig. 4). Based on comprehensive variant analysis using these data sets, the following filters were identified as those that reduced the number of false positives without excluding real mutations:

(a) Base substitutions: a Phred-scaled mapping quality of $>63$.

(b) Insertion: a minimum seven reads per site and at least $25 \%$ of reads had the extra nucleotides.

(c) Deletion: a minimum six reads per site and at least $30 \%$ of reads were missing the nucleotides.

\section{Detection of large INDELs and other chromosomal aberrations}

We also ran novel protocols (Jiang et al. 2011) to detect larger-scale variants such as insertions, inversions, and translocations. Novel codes were used to generate lists of "distant-pair" read pairs (where two paired reads ["mates"] align at unexpectedly distant regions of the reference sequence [progenitor]). Reads whose mates mapped $>750 \mathrm{bp}$ distant with respect to the reference (progenitor) were called, thus creating new (distant-pair) BAM files. Variant lists of covered regions (coverage $>5$ ) were generated and visually inspected with IGV. While no such mutations were detected in the six $\mathrm{FN} \mathrm{M}_{3}$ lines (E71, E99, E125, E128, E138, and E216 lines), analysis of a further $\mathrm{FN} \mathrm{M}_{3}$ line (E88) confirmed the reliability of these methods.

Further exhaustive searches were performed by systematically scanning each of the six $\mathrm{FN} \mathrm{M}_{3}$ line BAM files with IGV. These searches did not reveal any genomic variants (large or small) in FN lines additional to the 108 mutations initially identified by the above-described mutant detection procedures.

\section{Correction of FN-lineage mutation data with respect to spontaneous mutations}

A total of 108 homozygous variants (SBSs and INDELs) were identified in the genomes of six FN-irradiated $\mathrm{M}_{3}$ Arabidopsis lines. These variants are derived from a combination of both irradiation mutagenesis and also, to a far lesser extent, spontaneous mutational events. To determine the rate and spectrum of FN-induced mutations, the mutation rate and spectrum of spontaneously derived mutations needed to be deducted from our FN data. To do this, we used the results from the Col-0 MA lines experiment, which included a total of 98 substitutions, eight short deletions (ranging in size from 1 to $3 \mathrm{bp}$ ), four long deletions (ranging in size from 11 to $5445 \mathrm{bp}$ ), and five insertions (all $1 \mathrm{bp}$ ) across five MA lines over 30 generations (Ossowski et al. 2010).

To calculate the FN-specific mutation rate observed for single base variants in the different variant categories (substitutions and INDELs) and in the Ti/Tv profiles, we needed to correct the number of mutations observed in the $\mathrm{M}_{3}$ generation in each category twice; first for the spontaneous mutations that accumulated across six lines (E71, E99, E125, E128, E138, and E216) and second for the three generations of single seed descent from the reference strain (progenitor). To do this, we first calculated the frequencies of these events per MA line per generation, and then multiplied them by 18 ( $=6$ lines $\times 3$ generations) to get the correction factors for different variant categories. These numbers were then subtracted from the number of homozygous mutations observed in $\mathrm{M}_{3}$ generations (e.g., see Table $2 \mathrm{~A}$ ) to obtain the number of homozygous mutations arising as a result of FN treatment.

\section{Data access}

The Illumina DNA sequencing data files analyzed in this manuscript have been submitted to the NCBI Sequence Read Archive (SRA) (http://www.ncbi.nlm.nih.gov/sra) under accession number SRA051285.

\section{Acknowledgments}

This publication is based on work supported by Award No. KUKI1-002-03, made by King Abdullah University of Science and Technology (KAUST) and Biological and Biotechnological Sciences Research Council (BBSRC) grant no. BB/F020759/1. We thank the IGV group (Broad Institute, Cambridge, MA) for help with implementation of IGV. We thank David Buck and colleagues at the Wellcome Trust Centre for Human Genetics (Oxford, UK) Genomics Core for advice, help, and performing most of the genomic sequencing, supported by the Wellcome Trust Core grant no. 090532/ $\mathrm{Z} / 09 / \mathrm{Z}$.

\section{References}

Achard P, Cheng H, De Grauwe L, Decat J, Schoutteten H, Moritz T, Van Der Straeten D, Peng J, Harberd NP. 2006. Integration of plant responses to environmentally activated phytohormonal signals. Science 311: 91-94

Ahmad M, Jarillo JA, Smirnova O, Cashmore AR. 1998. Cryptochrome bluelight photoreceptors of Arabidopsis implicated in phototropism. Nature 392: 720-723.

Ang LH, Chattopadhyay S, Wei N, Oyama T, Okada K, Batschauer A, Deng XW. 1998. Molecular interaction between COP1 and HY5 defines a regulatory switch for light control of Arabidopsis development. Mol Cell 1: $213-222$.

Bowman JL, Smyth DR, Meyerowitz EM. 1991. Genetic interactions among floral homeotic genes of Arabidopsis. Development 112: 1-20.

Bruggemann E, Handwerger K, Essex C, Storz G. 1996. Analysis of fast neutron-generated mutants at the Arabidopsis thaliana HY4 locus. Plant J 10: 755-760.

Chen M, Chory J, Fankhauser C. 2004. Light signal transduction in higher plants. Annu Rev Genet 38: 87-117.

Copplestone D, Beresford N, Howard B. 2010. Protection of the environment from ionising radiation: Developing criteria and evaluating approaches for use in regulation. J Radiol Prot 30: 191-194.

Davis SJ, Kurepa J, Vierstra RD. 1999. The Arabidopsis thaliana HY1 locus, required for phytochrome-chromophore biosynthesis, encodes a protein related to heme oxygenases. Proc Natl Acad Sci 96: 65416546

Daya-Grosjean L, Sarasin A. 2005. The role of UV induced lesions in skin carcinogenesis: An overview of oncogene and tumor suppressor gene modifications in xeroderma pigmentosum skin tumors. Mutat Res 571: 43-56.

Friedberg EC, Walker GC, Siede W, Wood RD, Schultz RA, Ellenburger T. 2006. DNA repair and mutagenesis. ASM Press, Washington, DC.

Gan X, Stegle O, Behr J, Steffen JG, Drewe P, Hildebrand KL, Lyngsoe R, Schultheiss SJ, Osborne EJ, Sreedharan VT, et al. 2011. Multiple reference genomes and transcriptomes for Arabidopsis thaliana. Nature 477: 419423.

Greene EA, Codomo CA, Taylor NE, Henikoff JG, Till BJ, Reynolds SH, Enns LC, Burtner C, Johnson JE, Odden AR, et al. 2003. Spectrum of chemically induced mutations from a large-scale reverse-genetic screen in Arabidopsis. Genetics 164: 731-740.

Hinton TG, Alexakhin R, Balonov M, Gentner N, Hendry J, Prister B, Strand P, Woodhead D. 2007. Radiation-induced effects on plants and animals:

\section{Genome Research}


Findings of the United Nations Chernobyl Forum. Health Phys 93: 427440.

Hofer J, Turner L, Moreau C, Ambrose M, Isaac P, Butcher S, Weller J, Dupin A, Dalmais M, Le Signor C, et al. 2009. Tendril-less regulates tendril formation in pea leaves. Plant Cell 21: 420-428.

Hoffmann D, Jiang Q, Men A, Kinkema M, Gresshoff PM. 2007. Nodulation deficiency caused by fast neutron mutagenesis of the model legume Lotus japonicus. J Plant Physiol 164: 460-469.

Jander G, Baerson SR, Hudak JA, Gonzalez KA, Gruys KJ, Last RL. 2003. Ethylmethanesulfonate saturation mutagenesis in Arabidopsis to determine frequency of herbicide resistance. Plant Physiol 131: 139146.

Jiang C, Mithani A, Gan X, Belfield EJ, Klingler JP, Zhu J-K, Ragoussis J, Mott R, Harberd NP. 2011. Regenerant Arabidopsis lineages display a distinct genome-wide spectrum of mutations conferring variant phenotypes. Curr Biol 21: 1385-1390.

Kamra OP, Kamra SK, Nilan RA, Konzak CF. 1960. Radiation response of soaked barley seeds. Hereditas 46: 152-170.

Kawanishi S, Hiraku Y, Oikawa S. 2001. Mechanism of guanine-specific DNA damage by oxidative stress and its role in carcinogenesis and aging. Mutat Res 488: 65-76.

Kohchi T, Mukougawa K, Frankenberg N, Masuda M, Yokota A, Lagarias JC. 2001. The Arabidopsis HY2 gene encodes phytochromobilin synthase, a ferredoxin-dependent biliverdin reductase. Plant Cell 13: 425-436.

Korbel JO, Urban AE, Affourtit JP, Godwin B, Grubert F, Simons JF, Kim PM, Palejev D, Carriero NJ, Du L, et al. 2007. Paired-end mapping reveals extensive structural variation in the human genome. Science 318: $420-426$.

Ledger S, Strayer C, Ashton F, Kay SA, Putterill J. 2001. Analysis of the function of two circadian-regulated CONSTANS-LIKE genes. Plant J 26: 15-22.

Li H, Durbin R. 2010. Fast and accurate long-read alignment with BurrowsWheeler transform. Bioinformatics 26: 589-595.

Li X, Song Y, Century K, Straight S, Ronald P, Dong X, Lassner M, Zhang Y. 2001. A fast neutron deletion mutagenesis-based reverse genetics system for plants. Plant J 27: 235-242.

Li H, Ruan J, Durbin R. 2008. Mapping short DNA sequencing reads and calling variants using mapping quality scores. Genome Res 18: 1851-1858.

Li H, Handsaker B, Wysoker A, Fennell T, Ruan J, Homer N, Marth G, Abecasis G, Durbin R. 2009. The Sequence Alignment/Map format and SAMtools. Bioinformatics 25: 2078-2079.
Lin R, Wang H. 2004. Arabidopsis FHY3/FAR1 gene family and distinct roles of its members in light control of Arabidopsis development. Plant Physiol 136: 4010-4022.

Lunter G, Goodson M. 2011. Stampy: A statistical algorithm for sensitive and fast mapping of Illumina sequence reads. Genome Res 21: 936-939.

Lynch, M. Rate, molecular spectrum, and consequences of human mutation. Proc Natl Acad Sci 107: 961-968 (2010).

Martin B, Ramiro M, Martinez-Zapater JM, Alonso-Blanco C. 2009. A highdensity collection of EMS-induced mutations for TILLING in Landsberg erecta genetic background of Arabidopsis. BMC Plant Biol 9: 147. doi: 10.1186/1471-2229-9-147.

Müller HJ. 1928. The production of mutations by X-rays. Proc Natl Acad Sci 14: $714-726$.

Naito K, Kusaba M, Shikazono N, Takano T, Tanaka A, Tanisaka T, Nishimura M. 2005. Transmissible and nontransmissible mutations induced by irradiating Arabidopsis thaliana pollen with $\gamma$-rays and carbon ions. Genetics 169: 881-889.

Ossowski S, Schneeberger K, Lucas-Lledo JI, Warthmann N, Clark RM, Shaw RG, Weigel D, Lynch M. 2010. The rate and molecular spectrum of spontaneous mutations in Arabidopsis thaliana. Science 327: 92-94.

Prendergast JG, Campbell H, Gilbert N, Dunlop MG, Bickmore WA, Semple CA. 2007. Chromatin structure and evolution in the human genome. BMC Evol Biol 7: 72. doi: 10.1186/1471-2148-7-72.

Robinson JT, Thorvaldsdottir H, Winckler W, Guttman M, Lander ES, Getz G, Mesirov JP. 2011. Integrative genomics viewer. Nat Biotechnol 29: 2426.

Shikazono N, Suzuki C, Kitamura S, Watanabe H, Tano S, Tanaka A. 2005. Analysis of mutations induced by carbon ions in Arabidopsis thaliana. J Exp Bot 56: 587-596.

Somers DE, Sharrock RA, Tepperman JM, Quail PH. 1991. The hy3 long hypocotyl mutant of Arabidopsis is deficient in phytochrome B. Plant Cell 3: 1263-1274.

Sun TP, Goodman HM, Ausubel FM. 1992. Cloning the Arabidopsis Ga1 locus by genomic subtraction. Plant Cell 4: 119-128.

Viguera E, Canceill D, Ehrlich SD. 2001. Replication slippage involves DNA polymerase pausing and dissociation. EMBO J 20: 2587-2595.

Received September 2, 2011; accepted in revised form April 4, 2012. 


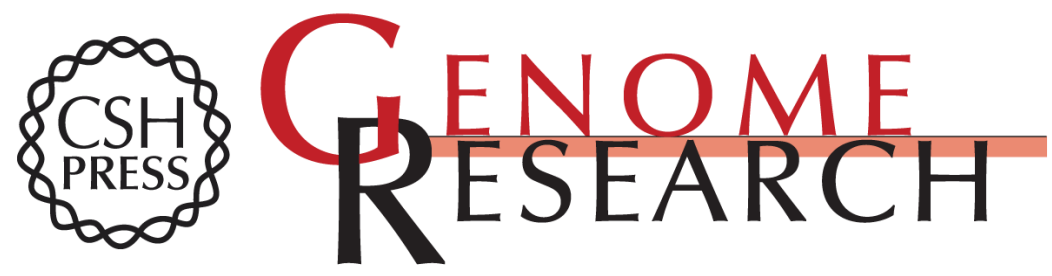

\section{Genome-wide analysis of mutations in mutant lineages selected following fast-neutron irradiation mutagenesis of Arabidopsis thaliana}

Eric J. Belfield, Xiangchao Gan, Aziz Mithani, et al.

Genome Res. 2012 22: 1306-1315 originally published online April 12, 2012

Access the most recent version at doi:10.1101/gr.131474.111

Supplemental
Material http://genome.cshlp.org/content/suppl/2012/04/06/gr.131474.111.DC1

References This article cites 37 articles, 15 of which can be accessed free at:

http://genome.cshlp.org/content/22/7/1306.full.html\#ref-list-1

Creative This article is distributed exclusively by Cold Spring Harbor Laboratory Press for the Commons

License first six months after the full-issue publication date (see

http://genome.cshlp.org/site/misc/terms.xhtml). After six months, it is available under a Creative Commons License (Attribution-NonCommercial 3.0 Unported License), as described at http://creativecommons.org/licenses/by-nc/3.0/.

Email Alerting Receive free email alerts when new articles cite this article - sign up in the box at the Service top right corner of the article or click here.

\section{Affordable, Accurate Sequencing.}

To subscribe to Genome Research go to:

https://genome.cshlp.org/subscriptions 